\title{
The Aesthetic Image of "Circle" in Chinese Classical Dance
}

\author{
Fan Yang
}

\author{
Art college of xiamen university 361005
}

\section{Keywords: circle; aesthetic image}

\begin{abstract}
Circle " is one of the morphological characteristics in Chinese classical dance. At the same time, it is also the presentation mode of the movement line. The pursuit of " circling " round Chinese classical dance in aesthetic image not only includes philosophical meaning, but also covers the national spirit. In order to trace the aesthetic characteristics of the " circle " and the characteristics of the national aesthetics, it is necessary to trace back to the historical context to see more clearly the reasons for the formation of this aesthetic feature.
\end{abstract}

\section{The "circle" of Chinese Classical Dance}

"Circle" is one of the morphological characteristics in Chinese classical dance, and it is also the presentation mode of the movement line. The content of " forming " into the Chinese classical dance teaching material implies all external, intuitive movements, gestures, connections between actions, transitional lines between gestures, and all visible forms and gestures. The process can be called " form. "[1] The " shape " here refers not only to the static state but also the course and trajectory in the course of the movement. So the morphological " circle " is reflected on the static dance posture as well as in the flow connection and action posture.

The embodiment of " circling " is manifested in posture and action lines, and emphasizes " circle shape ", " line circle ", and " big circle ". " Circular shape " means that the shape of the body follows a circular pattern, and the body lines are always rounded and smooth; the " circle circle " is the circular motion line, and the circle formed by the waist axis in the round circle, the vertical circle, and the eight-character circle. The law. " $\mathrm{Qu} "$ is a curve and a twist. In the body, it is expressed as a curve of the body line. It appears as a twist and it is like a dragon in the movement line.

Although the morphological characteristics of the " circle " were distilled from the opera and dance, the traditional opera dance evolved from the development of its historical history. For example, to trace the aesthetic characteristics of the " circle " and the characteristics of the national aesthetic, it is necessary to tracing back to the historical context, we can clearly see the reason for forming this aesthetic feature.

\section{Aesthetic Image of "Circle"}

The first appearance of "image" was in Liu Xie's "Wen Xin Diao Long", in which he made a very important analysis of aesthetic images. The word "imagery" can have two kinds of meanings. First, it refers to the imagery in the writer's mind (ie "the bamboo in the chest"); the first refers to the imagery embodied in the work (ie "the bamboo in the hands"). Liu Xie pointed out in his "Wen Xin Diao Long" that "The artisans of solitude, peeping at the imagery and transporting pounds," it indicate that Liu Xie's "imaging" refers to the first meaning.

The pursuit of "circle" in Chinese classical dance in aesthetic image not only includes philosophical meaning, but also covers the national spirit.

The Pythagorean School is the school of Western aesthetic history that first explored the essence of beauty. This school places emphasis on the study of the form of beauty. It believes that " the most beautiful of all plane figures are a circle, and the most beautiful of all three-dimensional circles are a sphere. " The Pythagorean school's interpretation of the beauty of " circling " tends to the formal 
beauty of the figure, and the significance and status of " circling " around Chinese national culture can be said to be deeply rooted in the flow of genes. "Circle " in Chinese classical aesthetics is not only rich in forms and carriers, but also has deep meaning and connotation.

In ancient China, due to the limited level of understanding and scientific and technological means, the earliest Chinese understanding of the universe was heaven and earth. In China's earliest extant mathematics and astronomy book " The Zhouyi Sutra ", there is the following statement : " The party belongs to the land, the heavens belongs to the heavens, and the heavens and the earth are round ". " Zhouyi justice " : " Taiji says that before the heavens and the earth are separated, the vital energy is mixed and " I Ching" will further symbolize the " circle of heaven and earth." ". Book of Changes " : " Easy to have tai cHi, is two students, two students and four students, four elephants gossip." " Yi Jing " in the yin and yang system of the world generated and its operating law derived from, as Circle is heaven, it is yang; Kun is land, square, yin. The first of all is the beginning of all things. High enticement : "round, plump.". The circle here is a complete and complete meaning.

As the earliest cosmological view on China, Tianyuan has also used the abstract connotation contained in the " circle " and " fang " extensively in architecture and art. Such as the Temple of Heaven, was built in the 18th year of Ming Yongle (1420). It was rebuilt by Emperor Qianlong and Emperor Guangxu of the Qing Dynasty. During the Me and Qing dynasties, the emperors were used to worship the imperial heavens and pray for a place of harvest. The Temple of Heaven is the general name for the two altars of Luqiu and Qigu. The altar walls are double, forming an inner and outer altar. The altar wall is a circle of the south and the north, symbolizing heaven and earth.

In Chinese classical garden architecture, the element of " circle " is omnipresent. The Dongmen is the most typical representative. It is divided into a gate on the fence, a gate in the park, and a partition gate in the park. The common forms are full moon, oval and rectangle. The gate plays a role in connecting the gardens. Among the gardens, the landscape is transferred from one landscape to the next. The connection between different areas is separated by a doorway, which not only allows the garden to maintain its circulation, but also distinguishes different landscapes. When people walk in the garden, their hearts are constantly Feeling " Do not have holes.". The flower windows in the garden are also quite pleasing, and they have a decorative effect in addition to air and light transmission. The style of the flower window is rich, with plants, animals and geometric composition as the main elements. The plant pattern uses " Mei Lan bamboo and chrysanthemum " to symbolize the noble quality theme, and the animal pattern is mainly the symbol of auspicious symbols and animal identity that highlights the identity. The geometric composition is divided into between a single geometric pattern and a composite geometric pattern, the single pattern are mostly round, square, and triangular, and the most common of the composite patterns is the outer inner circle. This element of " circling " frequently appears in garden architecture, not only reflects the love and persistence of " circle", It is not only a manifestation of the cosmological outlook that combines man and nature, but also a manifestation of the doctrine of golden mean philosophy.

The " circle " psychology is reflected on the ancient Chinese philosophy as the golden mean. " The Analects of Confucius - Yong Ye " also says : " The mean of the mean is the virtue, too. It is the highest ethical standard that treats people in terms of compromise, does not go to extremes, and people rarely implement it for a long time." ". The Golden Mean " clouds : " The emotions of joy, grief, sadness and joy are all in the midst of the middle festival.". [Hey] When the person's heart has no feelings of joy, anger, or sadness, it is called "middle, " and when emotion occurs, It is controlled so that it is still in the state of being maintained or restored. " The destiny of the destiny of nature, the precept of sexuality, and the preaching of Taoism.". The interpretive nature is endowed by nature. Following the nature is the Tao. Following the Taoism is self-education. " In the middle of the world, there is also a big book in the world, and also in the world. The same thing is true of/to the world. To neutrality, heavenly status, and all things are cultivated.". be the original face of everything in the universe ; and is the rule that all things are always following. When it reaches a level of neutrality, everything in heaven and earth will be returned to its place. Each of them will 
perform their duties, bringing everything into harmony, and unit each other. " Zhonghe " advocates the spirit of harmony between man and nature. " The Doctrine of the Mean " as an ancient Confucian philosophical view advocates the harmony between man and man, between heaven and self, where the idea of "'harmony " and the attribute of the geometric circle " circled " overlap each other. The philosophical view of Confucianism is in some form. The degree of reflection of the " round " forms into psychological performance.

The symbolism of the existence of "circle" in world outlook and philosophy is rooted in national culture, ideology and psychology. This gives the Chinese an infinite appreciation for "circle". In modern life, the element of "circle" is from The shape of the object to the meaning of things, or for the representative of nationality, is still more used as a cultural symbol of artistic design. It is not difficult to see why the Chinese classical dance chose "circle" as one of the morphological features. The round movement and round shape as the modality style emphasized by the national dance cover the aesthetic characteristics of the nation.

"Qu" and "circle" behave in similar ways. The curve is part of the "circle". The beauty of the lines represented by the curve also comes from the attachment to the "circle."

The "circle" in Chinese classical dance is closely related to ancient Chinese consciousness of universe and philosophy. The Tai Chi map is a manifestation of the idea of the Book of Changes.

"Easy" has tai chi and is a source of life." ( "Book of Changes" vol. VII, page 10) "Tai Chi” is the original of the universe, Tai Chi' s big circle means Tai Chi, "Tai Chi is born with two instruments, and two instruments are born with four images." Two kinds of devices refer to yin and yang. Four images refer to the four seasons of spring, summer, autumn and winter. They are expressed in terms of lunar, sun, Shaoyin, and Shaoyang. In the taiji diagram, the y-line separated by yin and yang fish, indicating that the two parts are independent of each other. The two small circles in the tai chi diagram represent yin and yang respectively, the yang fish have yin xiaoyin, and the yin fish contains yang fish, which symbolizes yin and yang. Yang and yin. If the eight-character circle is introduced horizontally into the Taiji Eight Diagrams for image analysis, it can be seen that 8 contains two opposite s, and the two opposite $\mathrm{s}$ and the mysterious curve of Yin and Yang in the center of the Taiji diagram are completely same. [5] From this, we also know the origin and basis of the eight-character circle, and the motion trajectory of the eight-character circle also contrasts the images of the universe that are contained in the Taiji diagram.

The waist, the most important part of the flat circle, the vertical circle, and the eight-character circle, uses the body as the media, and coordinates the coordination of the upper and lower body to assist the body of presenting three different circular movement trajectories. Take the vertical circle and the eight-character circle as an example, the formation of these two kinds of circular movement lines is shown in the dynamic process. It requires the twist of the waist to complete the complete circular route. If the waist does not twist, then this Both types of circles will not be formed.

Although the morphological characteristics of the "circle" are derived from studies and learning from traditional opera and martial arts, the formation and origin of these four morphological features are not limited to opera and martial arts, but are accumulated during the long historical development. To trace the causes of each morphological feature and the reasons that have been passed down from inheritance, we need to go back to the basis of history so that we can better understand the imagery contained in each morphological feature.

\section{References}

[1] Ye Lang, Outline of History of Chinese Aesthetics, Shanghai People's Publishing House

[2] Li Zehou's "The Course of Beauty", Tianjin Academy of Social Sciences Press

[3] Feng Youlan's "History of Chinese Philosophy" Chongqing Publishing House 
[4] Zong Baihua, “Aesthetic Walk” Shanghai People's Publishing House

[5] 5, Tang Mancheng, Jin Hao "Chinese classical dance body rhyme teaching method" Shanghai Music Publishing House

[6] Yuan He "Dance and Traditional Culture" Peking University Press

[7] Liao Qun and Yi Pingce “Chinese aesthetic culture stone - pre-Qin volumes" Shandong Pictorial Publishing House 